http://dx.doi.org/10.1590/1981-7746-sol00199

\title{
Pareceristas ad hoc do volume 16 (ano 2018)
}

Adelyne Maria Mendes Pereira / Fundação Oswaldo Cruz

Alcindo Antônio Ferla / Universidade Federal do Rio Grande do Sul

Alda Lacerda / Fundação Oswaldo Cruz

Aline Guerra Aquilante / Universidade Federal de São Carlos

Álvaro Roberto Crespo Merlo / Universidade Federal do Rio Grande do Sul

Ana Kalliny de Sousa Severo / Universidade Estadual de Campinas

Ana Laura Brandão / Fundação Oswaldo Cruz

Ana Lúcia Abrahão da Silva / Universidade Federal Fluminense

Ana Lúcia de Moura Pontes / Fundação Oswaldo Cruz

Ana Paula Müller de Andrade / Universidade Estadual do Centro-Oeste

Andreia Mendes dos Santos / Pontifícia Universidade Católica do Rio Grande do Sul

Áquilas Nogueira Mendes / Universidade de São Paulo

Áurea Maria Zöllner Ianni / Universidade de São Paulo

Bianca Antunes Cortes / Fundação Oswaldo Cruz

Bruno Rêgo Deusdará Rodrigues / Universidade do Estado do Rio de Janeiro

Bruno Ferrari Emerich / Universidade Estadual de Campinas

Carla Garcia Bottega / Universidade Estadual do Rio Grande do Sul

Carla Jorge Machado / Universidade Federal de Minas Gerais

Carlos Dimas Martins Ribeiro / Universidade Federal Fluminense

Carlos Henrique Assunção Paiva / Fundação Oswaldo Cruz 
Cátia Corrêa Guimarães / Fundação Oswaldo Cruz

Cláudia Du Bocage Santos Pinto / Universidade Federal de Mato Grosso do Sul Cláudia Elizabeth Abbês Baeta Neves / Universidade Federal Fluminense Debora Carvalho Ferreira / Universidade Federal de Viçosa

Décio Vieira da Rocha / Universidade do Estado do Rio de Janeiro Edineide Jezine Mesquita Araujo / Universidade Federal da Paraíba Edvânia Ângela de Souza Lourenço / Universidade Estadual Paulista Elaine Silva Miranda / Universidade Federal Fluminense Eliane de Fátima Almeida Lima / Universidade Federal do Espírito Santo Eliane Chaves Vianna / Fundação Oswaldo Cruz Elisabeth Meloni Vieira / Universidade de São Paulo Elisete Casotti / Universidade Federal do Rio de Janeiro Fabiana de Oliveira Silva Sousa / Fundação Oswaldo Cruz Fabiana Schneider Pires / Universidade Federal do Rio Grande do Sul Gabriela Spanghero Lotta / Universidade Federal do ABC Geovani Gurgel Aciole / Universidade Federal de São Carlos Gilmar José de Toni / Universidade Federal da Integração Latino-Americana Graciela Dutra Sehnem / Universidade Federal de Santa Maria Grayce Alencar Albuquerque / Universidade Regional do Cariri Guilherme Souza Cavalcanti de Albuquerque / Universidade Federal do Paraná Iale Falleiros Braga / Fundação Oswaldo Cruz 
Ide Gomes Dantas Gurgel / Fundação Oswaldo Cruz

Ingrid D’Ávilla / Fundação Oswaldo Cruz

Jacqueline Fiuza da Silva Regis / Universidade de Brasília

Jaqueline Martins / Ministério da Saúde

Jeanne de Souza Lima / Universidade Cândido Mendes

João José Batista de Campos / Universidade Estadual de Londrina

José Alcides Figueiredo Santos / Universidade Federal de Juiz de Fora

José Roberto Franco Reis / Fundação Oswaldo Cruz

Julián José Gindin / Universidade Federal Fluminense

Juliana Balbinot Hilgert / Universidade Federal do Rio Grande do Sul

Laura Cavalcanti de Farias Brehmer / Universidade Federal de Santa Catarina

Leandro Barbosa de Pinho / Universidade Federal do Rio Grande do Sul

Leda Aparecida Vanelli Nabuco de Gouvêa / Universidade Estadual do Oeste do Paraná

Letícia Batista da Silva / Fundação Oswaldo Cruz

Lisie Alende Prates / Universidade Federal do Pampa

Lislaine Aparecida Fracolli / Universidade de São Paulo

Lucilane Maria Sales da Silva / Universidade Estadual do Ceará

Márcia Silveira Ney / Prefeitura Municipal de Duque de Caxias

Márcia Valéria Guimarães Cardoso Morosini / Fundação Oswaldo Cruz

Marcio Wagner Camatta / Universidade Federal do Rio Grande do Sul

Marco Antonio Carvalho Santos / Fundação Oswaldo Cruz 
Marco Aurélio da Ros / Universidade Federal de Santa Catarina

Marco José de Oliveira Duarte / Universidade Federal de Juiz de Fora

Maria Cristina Cavaleiro / Universidade Estadual do Norte do Paraná

Maria de Fátima Duarte Martins / Universidade Federal de Pelotas

Maria do Socorro Veloso de Albuquerque / Fundação Oswaldo Cruz

Maria Eulina Pessoa de Carvalho / Universidade Federal da Paraíba

Maria Geralda Viana Heleno / Universidade Metodista de São Paulo

Maria Ligia Rangel Santos / Universidade Federal da Bahia

Maria Marta Nolasco Chaves / Universidade Federal do Paraná

Mariana Nogueira / Fundação Oswaldo Cruz

Marise Ramoa / Fundação Oswaldo Cruz

Marly Marques da Cruz / Fundação Oswaldo Cruz

Mônica Martins de Oliveira / Universidade Estadual de Campinas

Mônica Vieira / Fundação Oswaldo Cruz

Neise Deluiz / Fundação Oswaldo Cruz

Nuria Malajovich Munoz / Universidade Federal do Rio de Janeiro

Olinda Maria de Fátima Lechmann Saldanha / Universidade do Vale do Taquari

Otilia Maria Lúcia Barbosa Seiffert / Instituto Nacional de Estudos e Pesquisas Educacionais Anísio Teixeira

Paola Andressa Scortegagna / Universidade Estadual de Ponta Grossa

Paula Gaudenzi / Fundação Oswaldo Cruz 
Paulo Henrique de Almeida Rodrigues / Instituto de Medicina Social / Universidade do Estado do Rio de Janeiro

Paulo Henrique Novaes Martins de Albuquerque / Universidade Federal de Pernambuco

Petrônio José de Lima Martelli / Fundação Oswaldo Cruz

Pilar Belmonte / Fundação Oswaldo Cruz

Renata Maria Zanardo Romanholi / Universidade Estadual Paulista Júlio de Mesquita Filho

Roberta Carvalho / Ministério da Saúde

Roberta Gondim / Fundação Oswaldo Cruz

Rodrigo Caprio Leite de Castro / Universidade Federal do Rio Grande do Sul

Roger Keller Celeste / Universidade Federal do Rio Grande do Sul

Rogerio Miranda Gomes / Universidade Federal do Paraná

Rosemeire Aparecida Scopinho / Universidade Federal de São Carlos

Sandra Maria Gadelha de Carvalho / Universidade Federal do Ceará

Simone Santos Oliveira / Fundação Oswaldo Cruz

Sonia Maria Rummert / Universidade Federal Fluminense

Thiago Dias Sarti / Universidade Federal do Espírito Santo

Valeria Romano / Fundação Oswaldo Cruz

Vera Lucia Luiza / Fundação Oswaldo Cruz

Vera Marques / Fundação Oswaldo Cruz

Vitória Solange Coelho Ferreira / Universidade Estadual de Santa Cruz

Viviane Cristina Vieira / Universidade de Brasília

Wendell Ficher Teixeira Assis / Universidade Federal de Alagoas 